\title{
CRITICAL LENGTHS FOR SEMILINEAR SINGULAR PARABOLIC MIXED BOUNDARY-VALUE PROBLEMS
}

BY

\author{
C. Y. CHAN AND SHANNON S. COBB
}

University of Southwestern Louisiana, Lafayette, Louisiana

\section{Introduction. Let}

$$
\begin{aligned}
H u & \equiv u_{x x}-u_{t}, \\
\Omega & \equiv(0, a) \times(0, T), \\
\Gamma & \equiv([0, a] \times\{0\}) \cup(\{0\} \times(0, T)), \\
S & \equiv\{a\} \times(0, T),
\end{aligned}
$$

where $T \leq \infty$. Also, let $u$ be a solution of the problem:

$$
H u=-f(u) \text { in } \Omega, \quad u=0 \text { on } \Gamma, \quad u=0 \text { on } S,
$$

where $f(u)$ tends to infinity as $u$ approaches $c^{-}$for some positive constant $c$. The length $a^{*}$ is said to be the critical length for the problem (1.1) if $u$ exists globally for $a<a^{*}$, and for $a>a^{*}$ there exists a finite time $T$ such that

$$
\max \{u(x, t): 0 \leq x \leq a\} \rightarrow c^{-} \text {as } t \rightarrow T^{-} .
$$

This finite time $T$ is called the quenching time. In the special case that $f(u)=$ $(1-u)^{-1}$, Kawarada [9] showed that (1.2) occurred for $a>2^{3 / 2}$. Acker and Walter [2] showed that under appropriate conditions on the forcing term $f(u)$, there existed a unique critical length $a^{*}$ for the problem (1.1). This result was then extended to forcing terms of the type $g\left(u, u_{x}\right)$ by Acker and Walter [3], and to $h\left(x, u, u_{x}\right)$ by Chan and Kwong [7]. Results on the behavior of the solution of the problem (1.1) with $a=a^{*}$ were given by Levine and Montgomery [10]. Existence of the critical length $a^{*}$ and its determination by computational methods were given by Chan and Chen [4] for a more general parabolic singular operator; they studied the problem:

$$
L u=-(1-u)^{-1} \text { in } \Omega, \quad u=0 \text { on } \Gamma, \quad u=0 \text { on } S,
$$

where $L u \equiv H u+b u_{x} / x$ with $b$ a constant less than 1 ; in particular, $a^{*}=1.5303$ (to five significant figures) for $b=0$. Similar results were given by Chan and Kaper [6] for the problem:

$$
L u=-f(u) \text { in } \Omega, \quad u=0 \text { on } \Gamma, \quad u_{x}=0 \text { on } S .
$$

Received April 26, 1990.

The work of the first author was partially supported by the Board of Regents of the State of Louisiana under Grant LEQSF(86-89)-RD-A-11. 
This includes the problem (1.1) as a special case since the solution of that problem is symmetric with respect to the line $x=a / 2$. We refer to the papers of Chan and Chen [4] and Chan and Kaper [6] for the significance of the expression $L u$. Critical lengths for global existence of solutions for a coupled system of two semilinear parabolic equations subject to zero initial-boundary data were given by Chan and Chen [5]. Existence of the critical size for the multidimensional version of the problem (1.1) was studied by Acker and Kawohl [1].

The main purpose here is to study the critical length for the following problem:

$$
L u=-f(u) \text { in } \Omega, \quad u=0 \text { on } \Gamma, \quad B u=0 \text { on } S,
$$

where $B u \equiv u_{x}+k u$. Here, $b$ is a constant less than $1 ; k$ is a positive constant; $f$ is nondecreasing and continuously differentiable on $[0, c)$ for some constant $c$ such that $f(0)>0$; and $\lim _{u \rightarrow c^{-}} f(u)=\infty$. As in the papers by Chan and Chen [4] and Chan and Kaper [6], we assume existence of a solution $u$ before its quenching time. In the problem (1.3), $u$ attains its maxima with respect to $x$ at $x=a$; unlike the problem (1.1), the singular term $b u_{x} / x$ as well as the third boundary condition in our present problem destroys the symmetry of the solution $u$ about the line $x=a / 2$, and shifts the points where $u$ attains its maxima with respect to $x$ from the line $x=a / 2$. Thus, they make the problem more difficult both theoretically and numerically.

In Sec. 2, we establish existence of a critical length $a^{*}$, and give a computational method to determine $a^{*}$. In Sec. 3, a method is given to determine an upper bound of the quenching time for a given $a$ greater than $a^{*}$. An algorithm is given in Sec. 4 to compute $a^{*}$. For illustration, a numerical example is given by taking $f(u)$ to be $(1-u)^{-1}$.

2. Critical length. Let us first establish the following results.

LEMMA 1. Let $u$ be a solution of the problem (1.4).

(a) There exists at most one solution.

(b) The solution $u$ is positive in $\Omega \cup S$.

(c) The solution $u$ is a strictly increasing function of $t$ for each $x \in(0, a]$.

(d) There exists a curve $\phi(t)$ such that for each $t \in(0, T), u$ is strictly decreasing in $x$ on $(\phi(t), a]$, and nondecreasing in $x$ on $[0, \phi(t)]$, where $\phi(t) \in(0, a)$.

Proof. (a) Let $u_{1}$ and $u_{2}$ be two distinct solutions, and $w \equiv u_{1}-u_{2}$. Then by the mean value theorem,

$$
\left[L+f^{\prime}(\eta)\right] w=0 \text { in } \Omega,
$$

where $\eta$ lies between $u_{1}$ and $u_{2}$. Without loss of generality, let $w>0$ somewhere. Since $f^{\prime}(\eta)$ is bounded above, it follows from the strong maximum principle (cf. Protter and Weinberger [12, pp. 168-169, 172, and 175]) that $w$ attains its positive maximum somewhere on $S$. At this point, $w_{x}>0$ by the parabolic version of Hopf's lemma (cf. Protter and Weinberger [12, pp. 170-172]). This contradicts $B w=0$ on $S$. Thus, there exists at most one solution. 
(b) Since $f(0)>0$, we have $L u+f(u)<f(0)$. By the mean value theorem, $\left[L+f^{\prime}(\eta)\right] u<0$, where $\eta$ lies between $u$ and 0 . The assertion then follows from the strong maximum principle and the parabolic version of Hopf's lemma.

(c) For any $h>0$, let

$$
w(x, t)=u(x, t+h)-u(x, t) .
$$

By the mean value theorem, $\left[L+f^{\prime}(\eta)\right] w=0$, where $\eta$ lies between $u(x, t+h)$ and $u(x, t)$. Since $w(x, 0)>0$ for $0<x \leq a, w(0, t)=0$, and $B w=0$ on $S$, it follows from the strong maximum principle and the parabolic version of Hopf's lemma that $w>0$ on $\Omega \cup S$. The assertion is then proved.

(d) It follows from Lemma 1 (b) that $u_{x}(a, t)=-k u(a, t)<0$ for $0<t<T$; by the parabolic version of Hopf's lemma, $u_{x}(0, t)>0$ for $0<t<T$. For any fixed $t$ and any positive $x_{0}(\leq a)$ such that $u_{x}\left(x_{0}, t\right)<0$, it follows from the mean value theorem that for any positive $\epsilon \quad\left(\leq x_{0}\right)$,

$$
0<u(\epsilon, t)-u(0, t)=u_{x}(\eta, t) \epsilon \text { for some } \eta \in(0, \epsilon) .
$$

Thus for each $t(>0)$, there exists a point $x \in\left(0, x_{0}\right)$ such that $u_{x}(x, t)=0$.

Differentiating the differential equation in (1.4) with respect to $x$, we obtain

$$
\left(L+f^{\prime}(u)-b / x^{2}\right) u_{x}=0 .
$$

Let $G$ be the component containing $S$ such that $u_{x}<0$ in $G$. Since $G$ does not intersect the line $x=0$, it follows by applying the strong maximum principle that $G$ is simply connected with $u_{x}=0$ on $\partial G \cap \Omega$, where $\partial G$ denotes the boundary of $G$. If $u_{x}\left(x_{1}, t_{1}\right)<0$ somewhere in $\Omega \backslash G^{-}$, where $G^{-}$denotes the closure of $G$, then by the continuity of $u_{x}$, there exists a neighborhood $N$ of $\left(x_{1}, t_{1}\right)$ such that $u_{x}<0$ in $N$ and $u_{x}=0$ on $\partial N \cap(\Omega \backslash G)$, but this contradicts the strong maximum principle. Thus, $u_{x} \geq 0$ in $\Omega \backslash G^{-}$, and $\partial G \cap \Omega=\phi(t)$.

Let

$$
l U \equiv U^{\prime \prime}+\frac{b}{x} U^{\prime}, \quad \beta U \equiv U^{\prime}+k U .
$$

With slight modification of the proof of Theorem 3 of Chan and Kaper [6], we obtain the following result.

TheOREM 2. If $T=\infty$ and $u(x, t) \leq C<c$ for some constant $C$, then $u$ converges uniformly on $[0, a]$ from below to a solution $U$ of the singular nonlinear two-point boundary-value problem:

$$
l U=-f(U), \quad U(0)=0=\beta U(a) .
$$

Furthermore, $u<U$ in $(0, a] \times[0, \infty)$.

In order to show that beyond the critical length there exists a finite time $T$ such that (1.2) holds, the following result is crucial.

Theorem 3. $B u(x, t) \geq 0$ in $\Omega$.

Proof. For any $\epsilon \in(0, a)$, let

$$
\begin{aligned}
\Omega_{\epsilon} & \equiv(\epsilon, a) \times(0, T), \\
\Gamma_{\epsilon} & \equiv([\epsilon, a] \times\{0\}) \cup(\{\epsilon\} \times(0, T)) .
\end{aligned}
$$


Let $u_{\epsilon}$ denote the solution of the (regular) problem:

$$
\begin{gathered}
L u_{\epsilon}=-f\left(u_{\epsilon}\right) \quad \text { in } \Omega_{\epsilon}, \\
u_{\epsilon}=0 \quad \text { on } \Gamma_{\epsilon}, \quad B u_{\epsilon}=0 \quad \text { on } S .
\end{gathered}
$$

An argument as in the proofs of Lemma 1 (b) and (c) shows that $u_{\epsilon}>0$ in $\Omega_{\epsilon} \cup S$, and $u_{\epsilon}$ is a strictly increasing function of $t$ for each $x \in(\epsilon, a]$. It follows from the strong maximum principle and the parabolic version of Hopf's lemma that $u_{\epsilon}$ strictly increases as $\epsilon$ decreases. In particular, we have $0<u_{\epsilon}<u$ in $\Omega_{\epsilon}$. Let us differentiate (2.2) with respect to $x$, and denote the partial derivative of $u_{\epsilon}$ with respect to $x$ by $u_{\epsilon, x}$. We obtain

$$
\left[L+f^{\prime}\left(u_{\epsilon}\right)-b / x^{2}\right] u_{\epsilon, x}=0 \quad \text { in } \Omega_{\epsilon} .
$$

Now,

$$
u_{\epsilon, x}(x, 0)=0 \text { for } \epsilon \leq x \leq a .
$$

For any $\tau \in(0, T)$,

$$
u_{\epsilon, x}(\epsilon, t) \geq 0 \text { and } u_{\epsilon, x}(a, t)=-k u_{\epsilon}(a, t)<0 \text { for } 0<t<\tau .
$$

Let $\Omega_{\epsilon \tau} \equiv[\epsilon, a] \times[0, \tau]$. By the strong maximum principle, $u_{\epsilon, x}$ attains its negative minimum somewhere on $\Omega_{\epsilon \tau}$ at $x=a$. Since $u_{\epsilon}(a, t)$ increases as $t$ increases, it follows that $u_{\epsilon, x}(x, t) \geq-k u_{\epsilon}(a, \tau)$ on $\Omega_{\epsilon \tau}$. An argument as in the proof of Lemma 1(d) shows that there exists a curve $\psi(t)$ such that for each $t \in(0, T)$, $\psi(t) \in(\epsilon, a)$ and $u_{\epsilon}$ is strictly decreasing in $x$ on $(\psi(t), a]$ and nondecreasing in $x$ on $[\epsilon, \psi(t)]$. Thus for $x \in(\psi(\tau), a), B u_{\epsilon}(x, \tau)>0$. Because $u_{\epsilon}(x, \tau)>0$ for $x \in(\epsilon, \psi(\tau)], B u_{\epsilon}(x, \tau)>0$ there. Since $\tau$ is arbitrary, we have

$$
B u_{\epsilon}(x, t)>0 \text { in } \Omega_{\epsilon} .
$$

Since $u_{\epsilon}$ is bounded, $\lim _{\epsilon \rightarrow 0} u_{\epsilon}$ exists. Let us denote this limit by $Z$. Then in $\Omega_{\epsilon}$, $0<u_{\epsilon} \leq Z \leq u$ and $B Z \geq 0$.

To prove that $Z=u$, let $\sigma \in(\epsilon, a)$ and $u_{\sigma}$ be the unique solution of the (regular) problem:

$$
\begin{aligned}
& L u_{\sigma}=-f\left(u_{\sigma}\right) \text { in } \Omega_{\sigma}, \\
& u_{\sigma}(x, 0)=0, \quad B u_{\sigma}(\sigma, t)=u_{\epsilon}(\sigma, t), \quad \text { on } S .
\end{aligned}
$$

The adjoint $L^{*}$ (cf. Friedman $\left[8\right.$, p. 26]) of $L$ in $\Omega_{\sigma}$ is given by

$$
L^{*} v=v_{x x}-(b v / x)_{x}+v_{t}
$$

with adjoint boundary conditions (cf. Polozhiy [11, p. 413]) given by

$$
v(\sigma, t)=0=v_{x}(a, t)+(k-b / a) v(a, t) .
$$

Let $R^{*}(\xi, \tau ; x, t)$ denote its Green's function (cf. Friedman [8, pp. 82-84 and 155]). In Green's identity (cf. Friedman [8, p. 27]),

$$
v L u-u L^{*} v=\left(v u_{x}-u v_{x}+b u v / x\right)_{x}-(u v)_{t},
$$


let $u=u_{\epsilon}$ and $v(\xi, \tau)=R^{*}(\xi, \tau ; x, t)$. Let us integrate this over the domain $(\sigma, a) \times(0, t-\delta)$, where $\delta$ is a small positive constant less than $t$. By letting $\delta$ tend to zero, we obtain

$$
\begin{aligned}
u_{\epsilon}(x, t)= & \int_{0}^{t} \int_{\sigma}^{a} R^{*}(\xi, \tau ; x, t) f\left(u_{\epsilon}(\xi, \tau)\right) d \xi d \tau \\
& +\int_{0}^{t} R_{\xi}^{*}(\sigma, \tau ; x, t) u_{\epsilon}(\sigma, \tau) d \tau \text { in } \Omega_{\sigma} .
\end{aligned}
$$

Since $R^{*}(\xi, \tau ; x, t)>0$ for $(\xi, \tau) \in(\sigma, a) \times(0, t)$ (cf. Friedman [8, p. 84]), it follows that $R_{\xi}^{*}(\sigma, \tau ; x, t) \geq 0$. As $\epsilon$ decreases, $u_{\epsilon}$ and $f\left(u_{\epsilon}\right)$ are nondecreasing. By the monotone convergence theorem (cf. Royden [13, p. 84]),

$$
\begin{aligned}
Z(x, t)= & \int_{0}^{t} \int_{\sigma}^{a} R^{*}(\xi, \tau ; x, t) f(Z(\xi, \tau)) d \xi d \tau \\
& +\int_{0}^{t} R_{\xi}^{*}(\sigma, \tau ; x, t) Z(\sigma, \tau) d \tau \text { in } \Omega_{\sigma} .
\end{aligned}
$$

Thus, $L Z=-f(Z)$ in $\Omega_{\sigma}$. Since $\sigma$ is arbitrary, it follows that $L Z=-f(Z)$ in $\Omega$. Now, $Z(x, 0)=0$ and $B Z=0$ on $S$. From $0 \leq u_{\epsilon} \leq Z \leq u$ in $\Omega$, we have $Z(0, t)=0$. Since $u$ is unique, it follows that $u=Z$. From (2.3), $B u \geq 0$ in $\Omega$.

Let $u(x, t ; a)$ denote the solution $u(x, t)$ of the problem (1.4). Then for any positive constant $\alpha$, let $h$ be a nonnegative constant such that $h<\alpha$.

Theorem 4. If $\lim _{t \rightarrow \infty} u(\phi(t), t ; a)=c$, then there exists a finite time $T$ such that

$$
\max \{u(x, t ; a+\alpha): 0 \leq x \leq a+\alpha\} \rightarrow c^{-} \text {as } t \rightarrow T^{-} .
$$

Proof. Let us assume that there does not exist a finite time $T$ such that (2.4) holds. Let

$$
w(x, t)=u(x+h, t ; a+\alpha)-u(x, t ; a) .
$$

By the mean value theorem,

$$
\left[L+f^{\prime}(\eta)\right] w=0 \text { in } \Omega,
$$

where $\eta$ lies between $u(x+h, t ; a+\alpha)$ and $u(x, t ; a)$. By Theorem $3, B w \geq 0$ on $S$. Since $w(x, 0)=0$ and $w(0, t) \geq 0$, it follows from the strong maximum principle and the parabolic version of Hopf's lemma that $w \geq 0$ on $\Omega \cup S$. That is,

$$
u(x+h, t ; a+\alpha) \geq u(x, t ; a) \quad \text { on } \Omega \cup S .
$$

Let us choose positive numbers $\epsilon \quad(<c)$ and $t_{0}$ such that

$$
f(z) \geq \frac{8 \epsilon}{\alpha}\left(\frac{2}{\alpha}+\frac{2|b|}{\phi\left(t_{0}\right)+\alpha / 4}\right)+\alpha^{2}
$$

for $z \in[c-\epsilon, c)$ and $u\left(\phi\left(t_{0}\right), t_{0} ; a\right) \geq c-\epsilon$. Also, let

$$
E \equiv\left(\phi\left(t_{0}\right)+\alpha / 4, \phi\left(t_{0}\right)+\alpha\right) \times\left(t_{0}, \infty\right) .
$$

By assumption, $u(x, t ; a+\alpha)$ exists for all $t>0$, and hence $u(x, t ; a+\alpha)<c$ in $E$. From (2.5) and Lemma $1(\mathrm{c}), u(x, t ; a+\alpha) \geq c-\epsilon$ on the parabolic boundary $\partial E$ of $E$. Let

$$
z(x, t)=c-\epsilon+\left[x-\phi\left(t_{0}\right)-\alpha / 4\right]\left[\phi\left(t_{0}\right)+\alpha-x\right]\left(t-t_{0}\right) \text { in } E .
$$


On $\partial E, z=c-\epsilon$. By direct computation,

$$
\begin{aligned}
L z= & -2\left(t-t_{0}\right)+\frac{b}{x}\left\{2\left[\phi\left(t_{0}\right)-x\right]+5 \alpha / 4\right\}\left(t-t_{0}\right) \\
& -\left[x-\phi\left(t_{0}\right)-\alpha / 4\right]\left[\phi\left(t_{0}\right)+\alpha-x\right] .
\end{aligned}
$$

In the domain

$$
\left(\phi\left(t_{0}\right)+\alpha / 4, \phi\left(t_{0}\right)+\alpha\right) \times\left(t_{0}, t_{0}+8 \epsilon / \alpha^{2}\right),
$$

denoted by $D$, we have for $z \in[c-\epsilon, c)$,

$$
L z+f(z) \geq 0 \text { in } D \text {. }
$$

By the strong maximum principle, $u(x, t ; a+\alpha)>z$ in $D$. Since

$$
z\left(\phi\left(t_{0}\right)+\alpha / 2, t_{0}+8 \epsilon / \alpha^{2}\right)=c,
$$

it follows that

$$
u\left(\phi\left(t_{0}\right)+\alpha / 2, t_{0}+8 \epsilon / \alpha^{2} ; a+\alpha\right) \geq c .
$$

This contradiction proves the theorem.

We remark that Theorem 2 shows that there exists a critical length $a^{*}$ such that $u$ exists globally if $a<a^{*}$. This critical length is determined as the supremum of all $a$ for which a solution $U$ of the problem (2.1) exists; if $U\left(a^{*}\right)$ exists, then $u\left(a^{*}, t\right)$ exists also. Theorem 4 shows that (1.2) holds for some finite time $T$ when $a>a^{*}$.

To compute $a^{*}$, let us construct a sequence $\left\{U_{n}\right\}$ for $a<a^{*}$ by $U_{0}=0$ for $0 \leq x \leq a$, and for $n=1,2,3 \ldots$,

$$
l U_{n}+f\left(U_{n-1}\right)=0, \quad U_{n}(0)=0=\beta U(a) .
$$

In terms of Green's function $G(x ; \xi)$ corresponding to $l$, we have

$$
U_{n}(x)=\int_{0}^{a} \xi^{b} G(x ; \xi) f\left(U_{n-1}(\xi)\right) d \xi \text { for } n=1,2,3, \ldots,
$$

where

$$
G(x ; \xi)= \begin{cases}\left(1-q \xi^{1-b}\right) x^{1-b} /(1-b) & \text { for } 0 \leq x \leq \xi, \\ \left(1-q x^{1-b}\right) \xi^{1-b} /(1-b) & \text { for } \xi \leq x \leq a,\end{cases}
$$

with $q=k\left[(1-b) / a^{b}+k a^{1-b}\right]^{-1}$. The sequence is well defined. From (2.7) and the positivity of Green's function, $U_{n}(x)>0$ for $n \geq 1$ and $0<x \leq a$. Since $U_{n}^{\prime}(a)<0$, it follows that $U_{n}(x)$ attains its positive maximum somewhere in $(0, a)$. With slight modification of the proof of Theorem 5 of Chan and Kaper [6], we obtain the following result.

THEOREM 5. The sequence $\left\{U_{n}\right\}$ converges monotonically upwards to the minimal solution $U(<c)$ of the problem (2.1); furthermore,

$$
0<U_{n}<U_{n+1}<U, \quad 0<x \leq a, n=1,2,3, \ldots .
$$

The results established in the rest of this section are useful for computational purposes. To obtain an upper bound $a_{u}$ for $a^{*}$, let us use $U_{1}(x)$, which is a lower 
bound of the solution $U$ of the problem (2.1). From (2.7),

$$
\begin{aligned}
& U_{1}(x)=f(0)\left(\left(\frac{a^{1+b}}{1+b}-\frac{q a^{2}}{2}\right) \frac{x^{1-b}}{1-b}+\left(\frac{1}{2}-\frac{1}{1+b}\right) \frac{x^{2}}{1-b}\right) \text { for } b \neq-1, \\
& U_{1}(x)=f(0)\left(\frac{1-q a^{2}}{4} x^{2}+\frac{x^{2}}{2} \ln \frac{a}{x}\right) \quad \text { for } b=-1 .
\end{aligned}
$$

Differentiating (2.8) with respect to $x$ yields

$$
\begin{aligned}
& U_{1}^{\prime}(x)=f(0)\left(\left(\frac{a^{1+b}}{1+b}-\frac{q a^{2}}{2}\right) x^{-b}+\left(1-\frac{2}{1+b}\right) \frac{x}{1-b}\right) \text { for } b \neq-1, \\
& U_{1}^{\prime}(x)=f(0)\left(-\frac{q a^{2}}{2} x+x \ln \frac{a}{x}\right) \quad \text { for } b=-1,
\end{aligned}
$$

from which $U_{1}^{\prime}(x)=0$ occurs at

$$
\begin{aligned}
& x_{c}=\left\{\left[2 a^{1+b}-q a^{2}(1+b)\right] / 2\right\}^{1 /(1+b)} \text { for } b \neq-1, \\
& x_{c}=a e^{-q a^{2} / 2} \text { for } b=-1,
\end{aligned}
$$

where $U_{1}^{\prime \prime}=-f(0)<0$. This implies that the (absolute) maximum of $U_{1}(x)$ occurs at the value $x_{c}$. Thus, an upper bound $a_{u}$ for $a^{*}$ is determined by $U_{1}\left(x_{c}\right)=c$, which yields

$$
\begin{aligned}
& 2 a_{u}^{1+b}-q(1+b) a_{u}^{2}=2[2(1-b) c / f(0)]^{(1+b) / 2} \text { for } b \neq-1, \\
& 4 c=f(0) a_{u}^{2} e^{-k a_{u} /\left(2+k a_{u}\right)}\left[1+k a_{u} /\left(2+k a_{u}\right)\right] \text { for } b=-1 \text {. }
\end{aligned}
$$

To show that (2.9) determines exactly one $a_{u}$ for a given $b$, let us differentiate (2.8) with respect to $a$ :

$$
\begin{aligned}
& \frac{\partial U_{1}}{\partial a}=\frac{q^{2} f(0) x^{1-b}}{k^{2}}\left((1-b) a^{-b}+\frac{k^{2}}{2} a^{2-b}+\frac{k\left(2+b-b^{2}\right)}{2(1+b)} a^{1-b}\right) \quad \text { for } b \neq-1, \\
& \frac{\partial U_{1}}{\partial a}=\frac{f(0) x^{2}\left(4+3 k a+k^{2} a^{2}\right)}{2 a\left(4+4 k a+k^{2} a^{2}\right)} \text { for } b=-1 .
\end{aligned}
$$

In either case, $\partial U_{1} / \partial a>0$. Thus, $U_{1}$ increases as $a$ increases. Hence for a given $b, a_{u}$ is determined uniquely by (2.9). We obtain the following result.

LEMMA 6. $0<a^{*}<a_{u}$, where $a_{u}$ is determined uniquely by (2.9) for each given $b$.

Our next result is useful in stopping the computation of successive iterates.

LEMMA 7. For $0<x<a$, if $f^{\prime}$ is strictly increasing and $U_{n+1}-U_{n}>U_{n}-U_{n-1}$ for some positive integer $n$, then $U_{m+1}-U_{m}>U_{m}-U_{m-1}$ for $m=n+1, n+$ $2, n+3, \ldots$. 
Proof. The sequences $\left\{U_{n}\right\}$ and $\left\{f\left(U_{n}\right)\right\}$ are strictly increasing. For some $\eta$ between $U_{n+1}$ and $U_{n}$, and some $\zeta$ between $U_{n}$ and $U_{n-1}$, we have

$$
\begin{aligned}
U_{n+2}(x)-U_{n+1}(x) & =\int_{0}^{a} \xi^{b} G(x ; \xi)\left[f\left(U_{n+1}(\xi)\right)-f\left(U_{n}(\xi)\right)\right] d \xi \\
& =\int_{0}^{a} \xi^{b} G(x ; \xi) f^{\prime}(\eta)\left[U_{n+1}(\xi)-U_{n}(\xi)\right] d \xi \\
& >\int_{0}^{a} \xi^{b} G(x ; \xi) f^{\prime}(\zeta)\left[U_{n}(\xi)-U_{n-1}(\xi)\right] d \xi \\
& =U_{n+1}(x)-U_{n}(x) .
\end{aligned}
$$

The lemma then follows by using mathematical induction.

We now show that each iterate is a unimodal function.

LEMMA 8. For $a<a^{*}$, and each $n \geq 1$, the function $U_{n}(x)$ has a unique (positive) maximum.

Proof. Let $h$ be a critical point of $U_{n}(x)(n \geq 1)$ in the interval $(0, a)$. From (2.6),

$$
U_{n}^{\prime \prime}(h)=-f\left(U_{n-1}\right)<0,
$$

which shows that all critical points of $U_{n}(x)$ give relative maxima. Hence, there is exactly one (positive) maximum.

Since $l\left(U_{n+1}-U_{n}\right) \leq 0$, a proof similar to Lemma 8 gives the following result.

Lemma 9. For $a<a^{*}$ and each $n \geq 0$, the difference $U_{n+1}(x)-U_{n}(x)$ has a unique (positive) maximum.

3. Quenching time. To obtain an upper bound for the quenching time, we may consider the singular Sturm-Liouville problem:

$$
l w=-\lambda^{2} w, \quad w(0)=0, \quad \beta w(a)=0 .
$$

Its eigenvalues $\lambda^{2}$ are determined by

$$
\lambda J_{\nu-1}(\lambda a)+k J_{\nu}(\lambda a)=0,
$$

where $\nu=(1-b) / 2$ and $J_{\nu}(x)$ is the Bessel function of the first kind of order $\nu$. The eigenfunction corresponding to the smallest positive eigenvalue $\mu^{2}$ is $x^{\nu} J_{\nu}(\mu x)$. Following the argument of Sec. 4 of Chan and Kaper [6], the upper bound $t_{1}$ for the quenching time is determined by

$$
\left[\max _{0 \leq x \leq a} x^{\nu} J_{\nu}(\mu x)\right] g\left(t_{1}\right)=c,
$$

where $g(t)$ is given by the problem

$$
g^{\prime}(t)+\mu^{2} g(t)=G(g(t)), \quad g(0)=0 ;
$$

here,

$$
G(g(t)) \leq \inf \left\{\frac{f\left(x^{\nu} J_{\nu}(\mu x) g(t)\right)}{x^{\nu} J_{\nu}(\mu x)}: x \in[0, a]\right\}
$$


In particular, for $f(u)=(1-u)^{-1}$,

$$
\begin{aligned}
t_{1}= & \mu^{-1}\left(4-\mu^{2}\right)^{-1 / 2} \tan ^{-1}\left[\mu\left(4-\mu^{2}\right)^{-1 / 2}\right] \\
& -\left(2 \mu^{2}\right)^{-1} \ln \left[\left(4-\mu^{2}\right) / 4\right]+(\ln 2)\left(4-\mu^{2}\right)^{-1} .
\end{aligned}
$$

4. Numerical algorithm. By Lemma 6 , an upper bound $a_{u}$ of $a^{*}$ can be determined for each given $b$ by using the subroutine DZREAL (to find, to double precision, the real zeros of a real function using Muller's method) from the IMSL MATH/LIBRARY (Version 1.1, January, 1989; MALB-USM-PERFCT-EN8901-1.1). Since 0 can be taken as a lower bound of $a^{*}$, we can use the method of bisection to approximate $a^{*}$ by $a^{* *}=a_{u} / 2$. We use the representation formula (2.7) to compute $U_{n}(x)$ with $n \geq 1$ by using the following steps:

1. We divide the interval $\left[0, a^{* *}\right]$ into 20 equal subintervals with end points $x_{i}$ satisfying $0=x_{1}<x_{2}<x_{3}<\cdots<x_{21}=a^{* *}$.

2. At the 19 interior subdivision points, we evaluate

$$
y_{1}(x) \equiv x^{1-b} /(1-b), \quad y_{2}(x) \equiv\left(1-q x^{1-b}\right) /(1-b) ;
$$

we also compute $y_{2}\left(x_{21}\right)$. These values are stored in the memory of the computer for future use.

3. Let

$$
F_{n 1}(j, k)=\int_{x_{j}}^{x_{k}} \xi f\left(U_{n-1}(\xi)\right) d \xi, \quad F_{n 2}(j, k)=\int_{x_{j}}^{x_{k}}\left(\xi^{b}-q \xi\right) f\left(U_{n-1}(\xi)\right) d \xi .
$$

To save computer time, we evaluate $U_{n}\left(x_{11}\right)$ first. From (2.7),

$$
U_{n}\left(x_{11}\right)=y_{2}\left(x_{11}\right) F_{n 1}(1,11)+y_{1}\left(x_{11}\right) F_{n 2}(11,21) .
$$

To obtain $U_{n}\left(x_{10}\right)$, we only need to compute $F_{n 1}(10,11)$ and $F_{n 2}(10,11)$ since

$$
U_{n}\left(x_{10}\right)=y_{2}\left(x_{10}\right)\left[F_{n 1}(1,11)-F_{n 1}(10,11)\right]+y_{1}\left(x_{10}\right)\left[F_{n 2}(11,21)+F_{n 2}(10,11)\right] \text {. }
$$

In this way, we can successively compute $U_{n}$ at $x_{10}, x_{9}, x_{8}, \ldots, x_{2}$. Similarly,

$$
U_{n}\left(x_{12}\right)=y_{2}\left(x_{12}\right)\left[F_{n 1}(1,11)+F_{n 1}(11,12)\right]+y_{1}\left(x_{12}\right)\left[F_{n 2}(11,21)-F_{n 2}(11,12)\right] \text {. }
$$

Proceeding in this way, we obtain successively $U_{n}$ at $x_{12}, x_{13}, x_{14}, \ldots, x_{21}$.

To use a computer to calculate $U_{n}(x)$, we use three subroutines from the IMSL MATH/LIBRARY: DCSINT (to compute, to double precision, the cubic spline interpolant with the 'not-a-knot' condition) and DQDAG (to integrate, to double precision, a function using a globally adaptive scheme based on Gauss-Kronrod rules) with DCSVAL (to evaluate, to double precision, a cubic spline).

4. We use the subroutine DUVMGS (to find, to double precision, the minimum point of a nonsmooth (unimodal) function of a single variable) to determine $\max _{0 \leq x \leq a^{* *}} U_{n}(x)$ without any initial guesswork of where its critical point is since, by Lemma $8, U_{n}(x)$ is unimodal. Let us denote this maximum value by $M$.

5. We stop the computation of $U_{n}(x)$ as follows:

(a) If $M \geq c$, then $a^{* *}>a^{*}$.

(b) If $U_{n}-U_{n-1}>U_{n-1}-U_{n-2}$ for some $n$, then, by Lemma $7, a^{* *}>a^{*}$, provided $f^{\prime}$ is strictly increasing. 
(c) If $M<c$ and (by using Lemma 9)

$$
\max _{0 \leq x \leq a}\left[U_{n}(x)-U_{n-1}(x)\right]<5 \times 10^{-(r+1)}
$$

for some arbitrarily chosen nonnegative integer $r$, then $a^{* *}<a^{*}$. Here, $r$ determines the error tolerance in computing the successive iterates.

If $a^{* *}>a^{*}$, then we replace $a_{u}$ by $a^{* *}$; otherwise $u$ exists globally, and we replace 0 by $a^{* *}$. The above procedure of bisection is repeated until we reach the demanded accuracy (such as the difference between two successive approximations of $a^{*}$ is less than $\left.5 \times 10^{-(r+1)}\right)$. Since the difference between $a^{*}$ and the (ultimate) approximation $a^{* *}$ can be made as small as we like, this value $a^{* *}$ can be taken numerically to be $a^{*}$.

We apply the above algorithm to the case $f(u)=(1-u)^{-1}$ and $k=1$. We compute critical lengths $a^{*}$ for various given values of $b$ with the use of a computer. The results with $r=5$ are given in Table 1 .

TABLE 1. Critical lengths $a^{*}$ for four values of $b$.

\begin{tabular}{|cc|}
\hline$b$ & $a^{*}$ \\
0.40000 & 0.82415 \\
0.00000 & 0.99514 \\
-0.40000 & 1.14290 \\
-1.00000 & 1.33802 \\
\hline
\end{tabular}

Acknowledgment. The authors thank the referee for the helpful comment.

\section{REFERENCES}

[1] A. Acker and B. Kawohl, Remarks on quenching, Nonlinear Anal. 13, 53-61 (1989)

[2] A. Acker and W. Walter, The quenching problem for nonlinear parabolic differential equations, Lecture Notes in Math., Vol. 564, Springer-Verlag, New York, 1976, pp. 1-12

[3] A. Acker and W. Walter, On the global existence of solutions of parabolic differential equations with a singular nonlinear term, Nonlinear Anal. 2, 499-505 (1978)

[4] C. Y. Chan and C. S. Chen, A numerical method for semilinear singular parabolic quenching problems, Quart. Appl. Math. 47, 45-57 (1989)

[5] C. Y. Chan and C. S. Chen, Critical lengths for global existence of solutions for coupled semilinear singular parabolic problems, Quart. Appl. Math. 47, 661-671 (1989)

[6] C. Y. Chan and H. G. Kaper, Quenching for semilinear singular parabolic problems, SIAM J. Math. Anal. 20, 558-566 (1989)

[7] C. Y. Chan and M. K. Kwong, Existence results of steady-states of semilinear reaction-diffusion equations and their applications, J. Differential Equations 77, 304-321 (1989)

[8] A. Friedman, Partial Differential Equations of Parabolic Type, Prentice-Hall, Englewood Cliffs, NJ, 1964, pp. 26-27, 82-84 and 155

[9] H. Kawarada, On solutions of initial-boundary problem for $u_{t}=u_{x x}+(1-u)^{-1}$, Publ. Res. Inst. Math. Sci. 10, 729-736 (1975)

[10] H. A. Levine and J. T. Montgomery, The quenching of solutions of some nonlinear parabolic equations, SIAM J. Math. Anal. 11, 842-847 (1980)

[11] G. N. Polozhiy, Equations of Mathematical Physics, Hayden, New York, 1967, p. 413

[12] M. H. Protter and H. F. Weinberger, Maximum Principles in Differential Equations, SpringerVerlag, New York, 1984, pp. 168-172 and 175

[13] H. L. Royden, Real Analysis, 2nd ed., Macmillan, New York, 1968, p. 84 